\title{
Complicações vasculares e fatores relacionados a sua ocorrência após procedimentos hemodinâmicos percutâneos
}

\begin{abstract}
Vascular complications and factors related to their occurrence after percutaneous hemodynamic procedures
\end{abstract}

Complicaciones vasculares y factores asociados a su ocurrencia tras procedimientos hemodinámicos percutáneos

\section{Ana Carolina Pessoa Santos ${ }^{\mathrm{I}}$, Maria Letícia Bannwart Ambieli ${ }^{\mathrm{II}}$, Elaine Barros Ferreira ${ }^{\mathrm{III}}$, Priscilla Roberta Silva Rocha ${ }^{\text {IV }}$}

\begin{abstract}
Resumo: Objetivo: investigar as complicações vasculares em pacientes submetidos a procedimentos hemodinâmicos percutâneos e identificar fatores relacionados a sua ocorrência. Método: estudo retrospectivo em um hospital cardiológico, com pacientes que evoluíram com danos vasculares, entre janeiro de 2015 e dezembro de 2016. Os dados foram obtidos pela coleta em prontuários e analisados de forma descritiva e analítica. Resultados: foram incluídos 93 pacientes, a maioria idosos, do sexo masculino e portadores de hipertensão. A taxa de complicação vascular foi de $3 \%$, o hematoma local foi o mais frequente (86\%). O sexo feminino foi associado à ocorrência de danos vasculares mais graves como o hematoma retroperitoneal e o pseudoaneurisma $(\mathrm{p}=0,04)$. Conclusão: dentre as complicações vasculares, o hematoma foi o mais frequente e o sexo feminino foi fator preditor de risco, associado a injúrias mais graves. Reconhecer os fatores preditores de risco auxilia a assistência direcionada às necessidades individuais do paciente.
\end{abstract}

Descritores: Intervenção coronária percutânea; Assistência ao paciente; Hematoma; Enfermagem cardiovascular; Fatores de risco

Abstract: Objective: to investigate vascular complications in patients undergoing percutaneous hemodynamic procedures and identify factors related to their occurrence. Method: retrospective study conducted in a cardiology hospital with patients who had vascular damage between January 2015 and December 2016. Data were collected

\footnotetext{
I Enfermeira. Bacharel em Enfermagem, Universidade de Brasília, Faculdade de Ceilândia, Brasília, Distrito Federal, Brasil. E-mail: anacarol.pessoas@gmail.com, ORCID: https://orcid.org/0000-0003-3284-5414

II Enfermeira. Especialista em enfermagem cardiovascular, Hospital do Coração do Brasil, Brasília, Distrito Federal, Brasil. E-mail: maria.ambiel@hcbr.com.br. ORCID: https://orcid.org/0000-0001-8226-3848

III Enfermeira. Doutora em Enfermagem pela Universidade de Brasília, DF, Brasil. Professora Adjunta, Departamento de Enfermagem, Universidade de Brasília, Brasília, DF, Brasil. E-mail: elainebf@unb.br. ORCID: https://orcid.org/0000-0003-0428-834X

IV Enfermeira. Doutora em Ciências da Saúde pela Universidade de Brasília, DF, Brasil. Professora adjunta do curso de enfermagem Universidade de Brasilia - Faculdade de Ceilândia, Brasília, Brasil. E-mail: priscillarocha@unb.br. ORCID: https://orcid.org/0000-0002-2058-8548
} 
from medical records and submitted to descriptive analysis. Results: the study included 93 patients, most of them elderly, male and with hypertension. The rate of vascular complication was $3 \%$, local hematoma was the most common complication (86\%). Women were found to have more serious vascular injuries such as retroperitoneal hematoma and pseudoaneurysm than men $\operatorname{did}(\mathrm{p}=0.04)$. Conclusion: hematoma was the most frequent vascular complication, and female gender was a risk predictor associated with more serious injuries. Recognizing risk predictors contributes to the delivery of care tailored to the needs of each patient.

Descriptors: Percutaneous coronary intervention; Patient care; Hematoma; Cardiovascular nursing; Risk factors

Resumen: Objetivo: investigar las complicaciones vasculares en pacientes sometidos a procedimientos hemodinámicos percutáneos, así como identificar factores asociados a su ocurrencia. Método: estudio retrospectivo en hospital cardiológico, con pacientes que evolucionaron con lesiones vasculares, entre enero de 2015 y diciembre de 2016. Se obtuvieron los datos en prontuarios y se los analizaron de forma descriptiva y analítica. Resultados: participaron 93 pacientes, la mayor parte ancianos, del sexo masculino y portadores de hipertensión. La taja de complicación vascular fue de $3 \%$, el hematoma local fue el más frecuente (86\%). Se asoció al sexo femenino la ocurrencia de lesiones vasculares más graves como el hematoma retroperitoneal y pseudoaneurisma $(\mathrm{p}=0,04)$. Conclusión: entre las complicaciones vasculares, el hematoma fue el más frecuente y el sexo femenino fue factor predictor de riesgo, asociado a lesiones más graves. Reconocer los factores predictores de riesgo ayuda la asistencia a volverse a las necesidades individuales del paciente.

Descriptores: Intervención coronaria percutánea; Atención al paciente; Hematoma; Enfermería cardiovascular; Factores de riesgo

\section{Introdução}

As doenças cardiovasculares (DCV) apresentam alta incidência mundial, podendo acometer indivíduos em qualquer faixa etária. No Brasil, $20 \%$ dos óbitos registrados são decorrentes de DCV. ${ }^{1}$ A partir desse panorama, a Intervenção Coronariana Percutânea (ICP), procedimento para a visualização radiográfica dos vasos coronarianos após administração de contraste radiopaco, se tornou o processo hemodinâmico mais realizado no mundo como método de diagnóstico e de tratamento da Doença Arterial Coronária (DAC). ${ }^{2}$ Os avanços tecnológicos em intervenções invasivas permitiram não só o aumento do número de procedimentos, mas também a redução de custos, de complicações associadas e melhor acurácia terapêutica..$^{2-3}$

A ocorrência de complicações vasculares ${ }^{4}$ dependem da eficácia, do tipo de intervenção realizada, do acesso vascular escolhido e do tempo médio de duração do procedimento. ${ }^{5}$ Dentre 
estes, o hematoma na região de inserção do cateter é a mais comum. Este é caracterizado por extravasamento sanguíneo no interstício que pode evoluir para abaulamento local e compressão de estruturas adjacentes, diferente da equimose que ocorre por uma infiltração mínima de hemácias no interstício. ${ }^{6}$ Além do traumatismo decorrente da cateterização e do vasoespasmo, ainda podem ocorrer danos mais graves: o pseudoaneurisma (PSA) é caracterizado por um hematoma encapsulado ligado à artéria; já o hematoma retroperitoneal (HRP) é caracterizado por extravasamento sanguíneo a uma área mais distante, normalmente na região do flanco; ${ }^{7}$ e com menor frequência pode ocorrer isquemia distal ao sítio de punção, em decorrência de embolizações. $^{8}$

Apesar de relevantes, as lesões dos vasos não são frequentes (3,3\%), podendo ser assintomáticas, entretanto podem inviabilizar a reutilização da via vascular. ${ }^{6}$ Quando as injúrias acontecem, estão normalmente associadas a fatores vasculares locais como a calcificação da artéria puncionada, bem como a fatores sistêmicos como obesidade, idade, sexo, Hipertensão Arterial Sistêmica (HAS) e uso de anticoagulantes. ${ }^{9}$ Por isso, o encerramento vascular ao final do procedimento hemodinâmico, compreendido pela compressão manual e/ou por meio do uso de dispositivos de hemostasia, deve ser realizado com eficácia e efetividade. ${ }^{6-7,10}$

Estudos apontam que as consequências das complicações vasculares na ICP podem se equiparar às consequências do Infarto Agudo do Miocárdio (IAM), sendo associadas ao aumento da morbimortalidade, ao maior tempo de internação e a desfechos clínicos desfavoráveis. ${ }^{11}$ Dessa forma, é importante para o gerenciamento da qualidade de assistência de enfermagem o reconhecimento das características clínicas associadas à ocorrência de fragilidade do sistema cardiovascular, para que os pacientes em maior risco sejam identificados e estratégias preventivas sejam implementadas. $^{7}$ 
Assim, este estudo teve como objetivo investigar as complicações vasculares em pacientes submetidos a procedimentos hemodinâmicos percutâneos e identificar fatores relacionados a sua ocorrência.

\section{Método}

Estudo retrospectivo, realizado em uma Unidade de Cardiologia Intervencionista de um hospital cardiológico privado do Distrito Federal, Brasil, no ano de 2017. O hospital tem capacidade de 12 leitos de terapia intensiva, 30 leitos de internação, unidade ambulatorial, unidade de diagnóstico por imagem, centro de hemodinâmica, centro cirúrgico e medicina nuclear. A pesquisa foi desenvolvida de acordo com as diretrizes do STROBE. ${ }^{12}$

Foram incluídos pacientes maiores de 18 anos, submetidos a procedimentos hemodinâmicos por via percutânea entre janeiro 2015 e dezembro 2016 que evoluíram com desenvolvimento de alguma complicação vascular após o procedimento. Foram consideradas complicações: a formação de hematoma local, de HRP ou PSA. Pacientes com registros incompletos no prontuário foram excluídos da pesquisa.

Os dados secundários foram coletados a partir dos prontuários eletrônicos, por meio de instrumento semiestruturado elaborado pelos pesquisadores, o qual reuniu dados sociodemográficos e clínicos de cada paciente, a saber: identificação do paciente (registro eletrônico); variáveis demográficas (idade e sexo); variáveis clínicas (comorbidades preexistentes, medicamentos em uso, situação da alta); variáveis associadas ao procedimento hemodinâmico (sítio de punção, conduta clínica, exames de imagem utilizados para avaliar a extensão do hematoma, e/ou diagnóstico de PSA ou HRP) e o tempo de procedimento, sendo considerado o horário de início a partir da anestesia e o término a realização do curativo.

Os dados foram inseridos em planilhas do software Microsoft Office Excel e posteriormente tabulados e redirecionados ao programa estatístico Epinfo versão 7.2.2.6. A 
análise foi composta por duas etapas: (1) descritiva - caracterização dos pacientes incluídos por meio de cálculos de média, desvio padrão (DP) para as variáveis numéricas e de valores absolutos e percentuais, para as variáveis categóricas; (2) analítica - buscou-se identificar a associação das características estudadas (variáveis independentes/exposição) aos tipos específicos de complicações vasculares (variáveis dependentes/desfecho). A associação entre as complicações (desfecho) e as outras variáveis analisadas (exposição) foi representada pela razão de chances (odds ratios). Para a comparação entre os grupos (hematoma, HRP ou PSA) utilizou-se o teste Qui-quadrado para as variáveis categóricas e o teste $t$ de student para as variáveis numéricas. Foi considerado significativo valor de $\mathrm{p}<0,05$.

De acordo com os padrões éticos exigidos, o estudo foi aprovado pelo Comitê de Ética em Pesquisa da Fundação Oswaldo Cruz, sob parecer nº 1.709.243, Certificado de Apresentação de Certificação Ética 58249716.0.0000.8027, em 01 de setembro de 2016.

\section{Resultados}

Foram realizados 3120 procedimentos hemodinâmicos por via percutânea entre janeiro 2015 e dezembro 2016. Noventa e cinco pacientes evoluíram com pelo menos uma das complicações vasculares propostas na pesquisa (formação de hematoma local, de HRP ou PSA). Entretanto, 2 pacientes foram excluídos por dados incompletos no prontuário, assim 93 indivíduos foram considerados elegíveis para o estudo. A taxa de complicações vasculares foi de 3\%.

A idade média dos pacientes foi de 67,7 (DP=10,6) anos e houve predomínio de indivíduos do sexo masculino (56\%). A presença de comorbidades múltiplas foi frequente, 76,6\% dos pacientes incluídos tinham HAS e 47,3\% apresentaram algum tipo de dislipidemia. Apenas um paciente não fazia uso contínuo de algum medicamento (Tabela 1). 
Tabela 1 - Características clínicas e demográficas dos 93 pacientes com complicações vasculares decorrentes de procedimentos hemodinâmicos percutâneos. Brasília, DF, Brasil, 2017.

\begin{tabular}{lcc}
\hline \multicolumn{1}{c}{ Características } & $\begin{array}{c}\text { Frequência Absoluta } \\
\mathbf{n}=\mathbf{9 3}\end{array}$ & $\begin{array}{c}\text { Frequência relativa } \\
(\%)\end{array}$ \\
\hline Idade, Média (DP) & $67,7(10,6)$ & - \\
Sexo & & 56,0 \\
$\quad$ Masculino & 52 & 44,0 \\
Feminino & 41 & \\
Comorbidades & & 76,6 \\
$\quad$ Hipertensão arterial sistêmica & 72 & 47,3 \\
Dislipidemias & 44 & 35,4 \\
Outros & 33 & 33,0 \\
Diabetes melitus & 31 & 10,8 \\
Infarto agudo do miocárdio & 10 & 8,6 \\
Hipotireoidismo & 8 & 1,1 \\
Hipertireoidismo & 1 & \\
Medicamentos em Uso & & 69,9 \\
Antiplaquetário & 65 & 46,2 \\
Trombolítico & 43 & 39,8 \\
Anti-hipertensivos & 37 & 31,2 \\
Anti-lipidêmico & 29 & 31,2 \\
Outros & 29 & 18,3 \\
Antidiabético & 17 & 9,7 \\
Antitireoidianos & 9 & 6,5 \\
Anticoagulante & 6 & \\
\hline
\end{tabular}

O tempo médio da ICP foi de 67,5 (DP=54,6) minutos. A grande variação temporal decorreu do quadro clínico do paciente e da necessidade de nova intervenção hemodinâmica durante o procedimento. O local de punção mais frequente foi a artéria femoral, em $63,4 \%$ dos casos.

A ICP com fim diagnóstico ocorreu em $56 \%$ dos casos e com fim terapêutico em $44 \%$. Alguns pacientes realizaram mais de um procedimento hemodinâmico $(35,3 \%)$, sendo o cateterismo o mais frequente (41\%), seguido de angioplastia transluminal coronária (ATC) $(38,7 \%)$, angiografia $(4,7 \%)$ e outras intervenções $(15,5 \%)$. As demais técnicas realizadas são referentes à reserva do fluxo coronário (FFR), implante de endo prótese, filtro de veia cava, 
introdução de Shunt, estudo eletrofisiológico (EEF), aterectomia rotacional e tomografia de coerência óptica $(\mathrm{OCT})$.

Dentre as complicações vasculares avaliadas, 86\% desenvolveram hematoma local, caracterizada como complicação simples e 14\% desenvolveram injúrias mais graves, como HRP ou PSA (Tabela 2). Para investigar e/ou confirmar a ocorrência de HRP e PSA, 23 pacientes foram submetidos à realização de exame de imagem, 14\% realizaram Doppler e 10,8\% tomografia computadorizada. Os demais pacientes não apresentaram necessidade de investigação por meio de exame de imagem. A conduta implementada para o tratamento da complicação vascular predominantemente foi a conservadora em $98,9 \%$ dos casos. Em apenas um caso houve a necessidade de intervenção cirúrgica (Tabela 2).

Quanto ao desfecho clínico, $95,7 \%$ dos pacientes receberam alta hospitalar, 4,3\% apresentaram agravamento clínico e evoluíram para óbito, entretanto esse agravamento foi decorrente de complicações da doença de base e não do procedimento hemodinâmico (Tabela 2). Neste estudo não houve pacientes com registros de reinternação devido à complicação hemodinâmica. Os dados referentes às características relacionadas aos procedimentos hemodinâmicos são apresentados na Tabela 2.

Tabela 2 - Características Relacionadas aos Procedimentos Hemodinâmicos dos 93 pacientes com Complicações Vasculares. Brasília, DF, Brasil, 2017.

\begin{tabular}{lcc}
\hline \multicolumn{1}{c}{ Tipo de Procedimento } & Frequência Absoluta & Frequência relativa \\
& $\mathbf{n}=\mathbf{9 3}$ & $(\%)$ \\
\hline Tempo Procedimento (min), Média (DP) & $67,5( \pm 54,6)$ & - \\
Local de Punção & & 63,4 \\
$\quad$ Femoral & 59 & 32,3 \\
Radial & 30 & 2,2 \\
Ambos & 2 & 2,2 \\
Jugular & 2 & \\
Complicações Vasculares & & 86,0 \\
Hematoma & 80 & 8,6 \\
Hematoma retroperitoneal & 8 & 5,4 \\
Pseudoaneurisma & 5 &
\end{tabular}




\section{Conduta Terapêutica}

$\begin{array}{lcr}\text { Conservador } & 92 & 98,9 \\ \text { Cirurgia } & 1 & 1,1 \\ \text { Desfecho Clínico } & & \\ \text { Alta } & 89 & 95,7 \\ \text { Óbito } & 4 & 4,3\end{array}$

$\mathrm{Na}$ segunda etapa do estudo, foi avaliada a existência de fatores associados (variáveis de exposição) às complicações vasculares estudadas (variáveis desfecho). A tabela 3 mostra a distribuição das características analisadas para os dois grupos.

Tabela 3 -Fatores associados às complicações vasculares dos 93 pacientes, Brasília, DF, Brasil, 2017.

\begin{tabular}{|c|c|c|c|c|c|c|}
\hline \multirow[b]{2}{*}{$\begin{array}{l}\text { Características } \\
\text { (n) }\end{array}$} & \multicolumn{2}{|c|}{ Hematoma } & \multicolumn{2}{|c|}{$\mathrm{HRP}^{*}$ e $\mathrm{PSA}^{+}$} & \multirow[t]{2}{*}{$\mathrm{OR}^{*}(\mathrm{IC})$} & \multirow[t]{2}{*}{ p valor } \\
\hline & $\begin{array}{c}\text { Frequência } \\
\text { absoluta } \\
(\mathrm{n}=80)\end{array}$ & $\begin{array}{c}\text { Frequência } \\
\text { Relativa } \\
(\%)\end{array}$ & $\begin{array}{c}\text { Frequência } \\
\text { absoluta } \\
(\mathrm{n}=13)\end{array}$ & $\begin{array}{c}\text { Frequência } \\
\text { Relativa } \\
(\%)\end{array}$ & & \\
\hline Idade (anos) $\pm \mathrm{DP}$ & $67,7 \pm 10,35$ & - & $67,8 \pm 12,3$ & - & - & $0,46^{\S}$ \\
\hline \multicolumn{7}{|l|}{ Sexo (\%) } \\
\hline Feminino & 32 & 40 & 9 & 69,3 & & \\
\hline Masculino & 48 & 60 & 4 & 30,7 & $0,29(0,08-1,04)$ & $0,04^{\|}$ \\
\hline Presença & & & & & & \\
\hline \multicolumn{7}{|l|}{ Comorbidades (\%) } \\
\hline $\begin{array}{l}\text { Diabetes melitus } \\
(\operatorname{sim})\end{array}$ & 27 & 33,8 & 4 & 30,8 & $0,87(0,24-3,09)$ & $0,83^{\|}$ \\
\hline (não) & 53 & 66,2 & 9 & 69,2 & & \\
\hline HAS (sim) & 63 & 78,8 & 9 & 69,2 & $0,60(1,16-2,21)$ & $0,44^{\|}$ \\
\hline (não) & 17 & 21,2 & 4 & 30,8 & & \\
\hline Uso & & & & & & \\
\hline \multicolumn{7}{|l|}{ Medicamentos } \\
\hline Anticoagulante (sim) & 4 & 5,0 & 2 & 15,4 & $3,45(0,56-1,13)$ & $0,15^{\|}$ \\
\hline (não) & 76 & 95,0 & 11 & 84,6 & & \\
\hline $\begin{array}{l}\text { Antiplaquetário } \\
(\operatorname{sim})\end{array}$ & 57 & 71,2 & 8 & 61,6 & $0,64(0,19-2,18)$ & $0,47 \|$ \\
\hline (não) & 23 & 28,8 & 5 & 38,4 & & \\
\hline Trombolítico (sim) & 37 & 46,3 & 6 & 46,2 & $0,99(0,3-3,22)$ & $0,99 \|$ \\
\hline (não) & 43 & 53,7 & 7 & 53,8 & & \\
\hline Tempo (médio min) & $68,6 \pm 55,6$ & - & $60,8 \pm 49,5$ & - & - & $0,10^{\|}$ \\
\hline \multicolumn{7}{|l|}{ Desfecho } \\
\hline Alta & 78 & 97,5 & 11 & 84,6 & & \\
\hline Óbito & 2 & 2,5 & 2 & 15,4 & $0,14(0,01-1,10)$ & $0,03^{\S}$ \\
\hline
\end{tabular}


A idade, a presença de Diabetes Melitus (DM) e HAS, tempo de procedimento, bem como o uso de medicamentos que alteram a hemostasia (anticoagulantes, antiagregante plaquetários e trombolíticos) não foram significativamente associados à ocorrência de danos vasculares mais graves (HRP e PSA) ( $p>0,05)$, conforme Tabela 3. O sexo feminino foi associado a injúrias mais graves (HRP e PSA) ( $\mathrm{p}=0,04)$. Observou-se também que as complicações mais graves (HRP e PSA) foram associadas a um pior desfecho clínico (óbito) $(\mathrm{p}=0,03)$.

\section{Discussão}

Neste estudo, foram analisados pacientes que apresentaram complicação vascular após a realização de ICP, a fim de descrever sua ocorrência e os fatores associados a esse desfecho. A taxa de complicações (3\%) foi inferior à descrita na literatura..$^{9,13-14}$

A taxa de lesões vasculares encontrada na literatura oscila de $1 \%$ a $14 \%$, essa variação pode ser explicada pelas diferentes características clínicas (preditoras de complicações) de cada grupo avaliado. ${ }^{13} \mathrm{Um}$ estudo multicêntrico realizado no sul do Brasil com 2696 pacientes submetidos a procedimentos hemodinâmicos, relatou uma taxa de dano vascular geral de $8,8 \%{ }^{9}$

Dentre as complicações vasculares avaliadas nesta pesquisa, o hematoma local foi a condição mais frequente, o que comumente é visto em outros estudos..$^{9,15-16}$ A ocorrência do hematoma pode estar associada às características de risco do paciente, mas também pode ser influenciada por cuidados pós-procedimento, como a retirada do introdutor e a compressão mecânica da artéria, ${ }^{9,15-16}$ reforçando a importância de uma equipe de enfermagem capacitada para o cuidado hemodinâmico especializado. ${ }^{17}$

O HRP e o PSA são consideradas complicações vasculares mais complexas, ${ }^{16-17}$ e nesta pesquisa foram relatadas com menor frequência. A ocorrência de PSA $(5,4 \%)$ foi maior quando comparada a um outro estudo, que evidencia uma ocorrência de $2,6 \%{ }^{14}$ Do mesmo modo, a ocorrência de HRP foi superior a outro relato. ${ }^{18} \mathrm{O}$ HRP pode decorrer da difícil compressão 
mecânica, enquanto o PSA, devido a sua ligação direta com a artéria, pode estar associado à idade, sexo, presença de comorbidades, obesidade, tempo de procedimento, manipulação excessiva do vaso, bem como a calcificação da artéria., ${ }^{916-17}$

É sabido que a via femoral aumenta o risco de sangramento e complicações vasculares quando comparada com a via radial. ${ }^{8,11}$ Em uma pesquisa realizada no sul do Brasil, o uso da artéria femoral em ICP ocorreu em $95 \%$ dos casos. Neste estudo a via femoral foi utilizada em $60 \%$ dos procedimentos. A escolha frequente do acesso femoral pode ser explicada pela elevada presença de arteriosclerose e calcificação de vasos de pequeno e médio calibre em pacientes com idade avançada, uma vez que o uso do acesso radial implicaria em insucessos de acesso vascular, prolongando o tempo de procedimento, podendo aumentar a necessidade do uso de contraste no paciente, expondo-o a mais radiação e toxicidade farmacológica. ${ }^{19}$

A idade é um fator predisponente para ocorrência de danos vasculares. ${ }^{20}$ Pesquisas apontam que a idade entre 40 e 70 anos é fator de risco para lesões vasculares, dentre elas o sangramento, hematoma, HRP e PSA. ${ }^{20-22}$ Neste estudo, a média de idade foi $67,7( \pm 10,58)$ anos, caracterizando uma população idosa, contudo não se associou significativamente à gravidade da injúria vascular $(\mathrm{p}=0,46)$. A média de idade observada foi consonante com o contexto atual da pirâmide populacional, em que se percebe o aumento do envelhecimento e da expectativa de vida, maior incidência de DCV e do maior uso dos avanços tecnológicos terapêuticos. ${ }^{21}$

As complicações vasculares associadas à ICP são mais incidentes e graves no sexo feminino, ${ }^{22}$ o que pode ser justificado pela fragilidade cardiovascular decorrente das alterações hormonais relacionadas ao climatério e menopausa. ${ }^{23} \mathrm{Em}$ concordância ao encontrado na literatura, a frequência de complicações menos graves foi maior entre os homens, sendo o sexo masculino associado a uma menor chance de complicações graves (OR = 0,29; IC 95\% = 0,08-1,04; $p=0,04)$.

Em relação a presença de comorbidades, a HAS e o DM foram as comorbidades clínicas mais frequentes, entretanto não se apresentaram como fatores associados ao agravamento das 
injúrias vasculares $(\mathrm{p}=0,44 \mathrm{e} \mathrm{p}=0,83$ respectivamente). Todavia, sabe-se que as comorbidades podem estar relacionadas aos danos, pois são responsáveis por desencadear e/ou agravar o quadro do paciente, demandando o uso de alguns medicamentos como antiplaquetários e trombolíticos, os quais podem aumentar o risco de sangramento..$^{21,24-26}$

Sobre as medicações de uso contínuo, o Ácido Acetil Salicílico (AAS) foi o antiplaquetário de maior uso e durante a ICP o uso de trombolíticos foi frequente. Esses medicamentos são recomendados pela Diretriz Brasileira de Antiagregantes Plaquetários e Anticoagulantes em Cardiologia, contudo sabe-se que a dose-resposta varia de acordo com o risco de sangramento do paciente. A dupla antiagregação plaquetária é necessária para a realização da ICP, seu uso está associado ao maior risco de sangramento no pós-procedimento, de modo que, quando não puder ser evitado, medidas específicas devem ser tomadas nos cuidados ao paciente. ${ }^{27} \mathrm{O}$ uso desses medicamentos não foi significativamente associado ao agravamento das complicações vasculares $(p>0,05)$.

Assim, ao identificar sinais e sintomas sugestivos de complicações vasculares pós ICP, recomenda-se avaliação médica rápida e investigação clínica por meio de exames de imagem, quando necessário, para estabelecer a conduta terapêutica. ${ }^{17}$ Nesta pesquisa, o Doppler foi o exame mais utilizado para identificar e monitorizar a complicação. É um exame eficiente, tem baixo custo e pode ser realizado beira leito sem necessidade de anestesia ou contraste nefrotóxico, sendo excelente para diagnóstico e avaliação de complicações vasculares graves. ${ }^{28}$

Em relação ao desfecho final, a maioria dos pacientes evoluíram para alta hospitalar, o que condiz com o estudo a respeito do prognóstico de complicações vasculares quando identificadas e tratadas rapidamente..$^{20}$ Entretanto, os óbitos registrados na pesquisa não podem ser atribuídos à ocorrência de injúrias vasculares $(\mathrm{p}=0,03)$, mas sim a fatores como a doença base e agravamento do quadro clínico do paciente. Dados estes corroborados por achados da literatura, uma vez que o óbito não é um desfecho frequente relacionado ao dano vascular, mas 
sim a refratariedade ao tratamento da DCV base. ${ }^{20,29}$ Os dados apresentados e discutidos neste estudo reforçam a importância da avaliação da equipe de enfermagem no momento préprocedimento a fim de elencar fatores de risco existentes ou potenciais, ${ }^{28-29}$ e dessa forma propiciar um plano de cuidado e uma assistência qualificada e segura.

Foram identificadas como limitações do estudo a ausência de dados que permitissem a análise de características clínicas relevantes para o tema, a saber: dados relacionados ao introdutor (calibre), tempo de compressão após a retirada e dispositivo empregado (manual ou mecânico), bem como a ausência da estratificação de risco para sangramento.

\section{Conclusão}

A ocorrência de complicações vasculares após ICP ocorreu em 3\% dos pacientes, com maior frequência de hematomas $(86 \%)$. O sexo feminino foi associado a injúrias mais graves $(\mathrm{p}=$ $0,04)$ e estas foram associadas a um pior desfecho $(p=0,03)$.

De posse desses dados, percebe-se a necessidade de reconhecer os fatores preditores de risco de cada paciente para o desenvolvimento de complicações vasculares, auxiliando, assim, a equipe multiprofissional, em especial a equipe de enfermagem, a prover uma assistência direcionada às necessidades individuais do paciente. Embora os dados deste estudo reflitam características de uma população específica, estes podem oferecer subsídios para novas pesquisas.

\section{Referências}

1. Mansur AP, Favarato D. Tendências da taxa de mortalidade por doenças cardiovasculares no Brasil, 1980-2012. Arq Bras Cardiol [Internet] 2016 [acesso em 2018 out 20]; 0-0: 20-25. doi: 10.5935/abc.20160077

2. Conejo F, Ribeiro HB, Spadaro AG, Godinho RR, Faig SM, Gabrilaitis C, Okada MY et al. Segurança e preditores de sucesso da alta hospitalar no mesmo dia após intervenção coronária 
percutânea eletiva. Rev Bras Cardiol Intensiva [Internet] 2015 [acesso em 2018 out 20]; 23(1): 42-47. doi: doi.org/10.1016/j.rbci.2015.01.001

3. Mennuni MG, Pagnotta PA, Stefanini G G.Coronary Stents: The Impact of Technological Advances on Clinical Outcomes. Annals of Biomedical Engineering [internet]. 2015 [acesso em 2020 mar 17]; 44(2): 488-496. doi:10.1007/s10439-015-1399-z.

4. Sá CFR, Lobo JRP, Pereira AJM, Almeida MCC. Intervenção coronária percutânea - abordagem femoral vs abordagem radial. Revista Cardiopulmonar. Portugal [internet]. 2015 [acesso em 2018 mai 5]: 0-0: 25-30. Disponível em: http://hdl.handle.net/10400.11/2805

5. Aguiar BF, Rinaldi ECA, Cintho LMM, Martins CLS, Zimmerman MH. Importância dos cuidados de enfermagem no cateterismo cardíaco. Cienc Cuid Saude [internet]. 2016 Jul/Set [acesso em 2020 mar 20]; 15(3): 460-465. doi: 10.4025/cienccuidsaude.v15i3.24894

6. Andrade PB de, Rinaldi FS, Bienert IR de C, Bergonso MH, Matos MP et al. Fatores de risco para complicações relacionadas ao acesso vascular em pacientes submetidos à estratégia invasiva precoce. Rev Bras Cardiol Invasiva [internet]. 2015 [Acesso em 2017 mai 10]; 23(4): 242-246. doi: https://doi.org/10.1016/j.rbci.2016.12.001

7. Aoun J, Hattar L, Dgaily K, Wong G, Bhat T. Update on complications and their management during transradial cardiac catheterization. Expert Review of Cardiovascular Therapy [internet]. 2019 [acesso em 2020 mar 20]; 0-0: 1-34. doi: 10.1080/14779072.2019.1675510

8. Piva CD, Vaz E, Moraes MA, Goldmeyer S, Linch GFC, Souza EN. Desconfortos relatados pelos pacientes após cateterismo cardíaco pelas vias femoral ou radial. Rev Bras Cardiol Intensiva [internet]. Porto Alegre, 2014 [acesso em 2018 out 20]; 22(1):36-40. doi: 10.1590/0104-1843000000008.

9. Paganin AC, Beghetto MG, Feijo MK, Matte R, Sauer JM, Rabelo-Silva ER. Vascular complications in patients who underwent endovascular cardiac procedures: multicenter cohort study. Rev. Latino-Am. Enfermagem [internet]. 2018 [acesso em 2019 mar 17]; 26(e3060): 1-7. doi: http://dx.doi.org/10.1590/1518-8345.2672.3060.

10. Córdova ESM, Santos LR, Toebe D, Moraes MAP, Souza EN. Incidence of hemorrhagic complications with use of a radial compression device: a cohort study. Rev Esc Enferm USP [internet]. 2018 [acesso em 2020 jul 15];52(e03410): 1-6. DOI: http://dx.doi.org/10.1590/S1980-220X2017041003410

11. Silva BS, Mangione FM, Wili LF, Mauro MF, Cristóvão SA, Mangione JA. Análise dos pacientes submetidos à intervenção coronária percutânea por via radial. Dados do Registro SAFIRA. J Transcat Interven. [internet] 2018 [acesso em 2020 jul 15]; 26(1):eA0018. doi: https://doi.org/10.31160/ JOTCI2018;26(1)A0018

12. Cuschieri S. The STROBE guidelines. Saudi J Anaesth [internet] 2019 Apr [acesso em 2020 jul 15]; 13 (Suppl 1): S31-S34. doi: 10.4103/sja.SJA_543_18 
13. Zanatta LG, Cardoso O, Mota FM, Conti EP, Diehl D, Rodrigues APR. et al. Preditores e Incidência de Complicações Vasculares Após a Realização de Intervenções Coronárias Percutâneas: Achados do Registro IC-FUC. Rev Bras Cardiol Invas [internet]. 2008 [acesso em 17/03/20];16(3):301-306. doi: https://doi.org/10.1590/S2179-83972008000300010.

14. Brito Jr. FS, Magalhães MA de, Nascimento TCDC, Amorim IMG, Almeida BO, Abizaid A et al. Incidência e Preditores Contemporâneos de Complicações Vasculares após Intervenção Coronária Percutânea. Rev Bras Cardiol Invas [internet]. 2007 [acesso em 2020 mar 17]; 15(4): 394-399. doi: https://doi.org/10.1590/S2179-83972007000400014

15. Matte R, Hilário TSM, Reich R, Aliti GB, Silva ERR. Redução do repouso de cinco para três horas não aumenta complicações após cateterismo cardíaco: THREE CATH Clinical Trial. Rev Latino-Ame. Enfermagem [internet]. 2016 [acesso em 2019 feb 05]; 24: e2797. doi: http://dx.doi.org/10.1590/15188345.0725.2796.

16. Santos AFS, Silva IB, Carvalho SQ, Andrade Junior CV. Assistência de enfermagem a pacientes submetidos à angioplastia coronária: uma revisão de literatura. Ciências Biológicas e de Saúde Unit [internet]. 2017 [acesso em 2019 mar 10]; 4(1): 191-201. Disponível em: https://periodicos.set.edu.br/index.php/fitsbiosaude/article/view/4063/2386

17. Reich R, Rabelo-Silva ER, Santos SM, Almeida MA. Complicações do acesso vascular em pacientes submetidos a procedimentos percutâneos em hemodinâmica: revisão de escopo. Rev Gaúcha Enferm [internet]. 2017 [acesso em 2020 mar 19];38(4):e68716. doi: http://dx.doi.org/10.1590/19831447.2017.04.68716.

18. Régis AP, Rosa GCD, Lunelli T. Cuidados de enfermagem no cateterismo cardíaco e angioplastia coronariana: desenvolvimento de um instrumento. Revista Recien [internet]. 2017 [acesso em 2020 mar 16]; 7(21):3-20. Disponível em: https://www.recien.com.br/index.php/Recien/article/view/238

19. Zukowski CN, Wozniak I, Souza Filho NFS de, Cordeiro EA, Rell A, Leal M et al. Acesso radial vs femoral em pacientes com idade avançada submetidos à intervenção coronária percutânea. Rev Bras Cardiol Intensiva [internet]. 2014 [acesso em 2017 jul 23]; 22(2):125-30. doi: http://dx.doi.org/10.1590/01041843000000022.

20. Leão AMO, Vilagra MM. Perfil dos pacientes submetidos à intervenção coronariana percutânea no serviço de hemodinâmica do hospital universitário sul fluminense, Vassouras -RJ. Revista de Saúde [internet], 2012 [acesso em 2018 nov 25]; 3(1): 27-32. doi: https://doi.org/10.21727/rs.v3i1.78.

21. Aguiar BF, Rinaldi ECA, Cintho LMM, Martins CLS, Zimmerman MH. Importance of nursing care in cardiac catheterization. Cienc Cuid Saúde [internet]. 2016 [acesso em 2017 set 15]; 15(3): 460-465. doi: 10.4025/cienccuidsaude.v15i3.24894. 
22. Correia LCL, Ferreira F, Kalil F, Silva A, Pereira L, Carvalhal M et al. Comparação entre os escores ACUITY e CRUSADE para predição de sangramento maior na síndrome coronariana aguda. Arq Bras Cardiol [internet]. 2015 [acesso em 2019 jun 01]; 105(1):20-27. doi: http://dx.doi.org/10.5935/abc.20150058.

23. Vila KM, Rocha RG, Naves CBOC, Almeida LF, Marta CB, Oliveira CSR. Clinicalepidemiological profile of patients undergoing cardiac catheterization procedures at a university hospital in Rio de Janeiro state. Rev Fun Care [internet]. 2019 jul/set [acesso em 2020 mar 17]; 11(4):894899. doi: http://dx.doi.org/10.9789/2175-5361.2019.v11i4.894-899.

24. Kuhn O, Bueno JF, Loro M, Bernat KA, Winkelmann E, Rosanelli C. Perfil de pacientes submetidos à cateterismo cardíaco e angioplastia em um hospital geral. Revista Contexto \& Saúde [interntet] 2015 [acesso em 2020 jul 15]; 15(29): 4-14.doi: https://doi.org/10.21527/2176-7114.2015.29.4-14

25. Lima MSM, Dantas RAN, Mendes NPN, Alves LCM, Silva TTM, Brito AGR, et al. Clinicalepidemiological aspects of patients submitted to Percutaneous Coronary Intervention in a university hospital. Rev Bras Enferm [Internet]. 2018 [acesso em jul 15];71(6):2883-90. doi: http://dx.doi.org/10.1590/0034-7167-2018-0012

26. Sociedade Brasileira de Cardiologia/ Sociedade Brasileira de Hipertensão/ Sociedade Brasileira de Nefrologia. VII Diretriz brasileira de hipertensão. Arq Bras Cardiol [internet] 2016 [acesso em 2020 jul 15]; $\quad 107 \quad(3, \quad$ Sup $\quad 3): \quad$ 1-103. Disponível em: http://publicacoes.cardiol.br/2014/diretrizes/2016/05_HIPERTENSAO_ARTERIAL.pdf

27. Serrano Jr. CV, Soeiro AM, Leal TCAT, Godoy LC, Biselli B, Hata LA et al. Posicionamento sobre Antiagregantes Plaquetários e Anticoagulantes em Cardiologia - 2019. Arq Bras Cardiol [internet] 2019 [acesso em 2020 jul 15]; 113(1):111-134. Diponível em: http://publicacoes.cardiol.br/portal/abc/portugues/2019/v11301/pdf/11301024.pdf

28. Stolt M, Braun-Dullaeus R, Herold J. Do not underestimate the femoral pseudoaneurysm. Vasa. [internet] 2018 [acesso em jul 15];47(3):177-185. doi:10.1024/0301-1526/a000691

29. Deça Júnior A, Brito MVB, Rodrigues LS, Martins RJS, Rabelo PPC. Check list da visita préoperatória de enfermagem: avaliação da qualidade dos dados. Rev. Enferm. UFSM - REUFSM Santa Maria, RS [internet]. 2020 [acesso em 2020 mai 27]; 10(22): 1-13, 2020. doi: 10.5902/2179769236082

Editora Científica: Tânia Solange Bosi de Souza Magnago

Editora Associada: Alexa Pupiara Flores Coelho

Fomento/Agradecimento: Agradecemos ao Hospital do Coração do Brasil pela disponibilidade de campo de pesquisa. 


\section{Autor correspondente}

Priscilla Roberta Silva Rocha

E-mail: priscillarocha@unb.br

Endereço: Faculdade de Ceilândia, Universidade de Brasília, Campus s/n, Centro Metropolitano, Brasília, Distrito Federal, Brasil, CEP: 72220-275

\section{Contribuições de Autoria}

1 - Ana Carolina Pessoa Santos

Concepção ou desenho do estudo/pesquisa, análise e/ou interpretação dos dados, revisão final com participação crítica e intelectual no manuscrito

\section{2 - Maria Letícia Bannwart Ambiel}

Concepção ou desenho do estudo/pesquisa; (3) revisão final com participação crítica e intelectual no manuscrito

\section{3 - Elaine Barros Ferreira}

Análise e/ou interpretação dos dados (3) revisão final com participação crítica e intelectual no manuscrito

\section{4 - Priscilla Roberta Silva Rocha}

Concepção ou desenho do estudo/pesquisa, análise e/ou interpretação dos dados, revisão final com participação crítica e intelectual no manuscrito

\section{Como citar este artigo}

Santos ACPS, Ambiel MLB, Ferreira EB, Rocha PRS. Complicações vasculares e fatores relacionados a sua ocorrência após procedimentos hemodinâmicos percutâneos. Rev. Enferm. UFSM. 2020 [Acesso em: Anos Mês Dia]; vol.10, e90: 1-16. DOI:https://doi.org/10.5902/2179769241286 